\title{
Pumping in quantum dots and non-Abelian matrix Berry phases
}

\author{
N.Y. Hwang, S.C. Kim, P.S. Park, and S.-R. Eric Yang* \\ Physics Department, Korea University, Seoul Korea
}

(Dated: November 4, 2018)

\begin{abstract}
We have investigated pumping in quantum dots from the perspective of non-Abelian (matrix) Berry phases by solving the time dependent Schrödinger equation exactly for adiabatic changes. Our results demonstrate that a pumped charge is related to the presence of a finite matrix Berry phase. When consecutive adiabatic cycles are performed the pumped charge of each cycle is different from the previous ones.
\end{abstract}

PACS numbers: 71.55.Eq, 71.70.Ej, 03.67.Lx, 03.67.Pp

\section{INTRODUCTION}

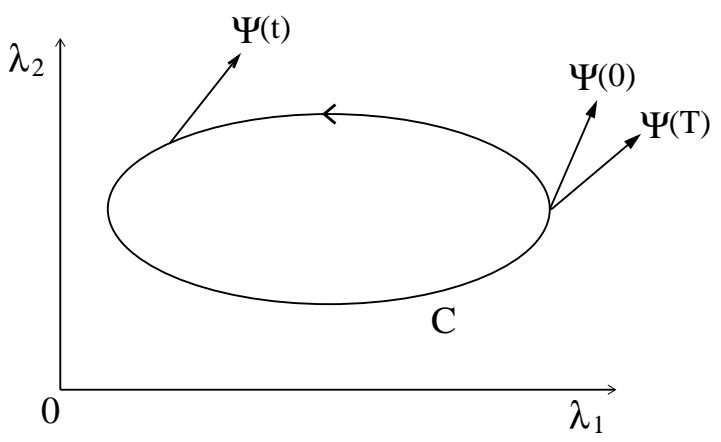

FIG. 1: Adiabatic path in the parameter space is shown. An electron is in the state $|\Psi(0)\rangle$ initially. After the pumping action it is in the final state $|\Psi(T)\rangle$, where $T$ is the period of the cycle. The initial and final states $|\Psi(0)\rangle$ and $|\Psi(T)\rangle$ are related to each other through a matrix Berry phase. During the adiabatic cycle the electron state does not leave the doubly degenerate Hilbert subspace.

A semiconductor quantum dot electron pump [1, 2] is a device that can make a dc current flow in unbiased systems. In such a device a charge can be pumped through the dot when the shape of the double barrier potential undergoes an adiabatic cyclic change. Quantum pumping is a fascinating subject and considerable experimental [3, 4, 5, 5] and theoretical studies have been carried out $7,18,19,10,11,12,13,14,15,16,17,18$, 19, 20]. Recently effects of noise and decoherence 21, $22,23,24]$, interactions [25, 26, 27, 28, 29, 30, 31], and spin [6, 32, 33, 34] have been investigated. The pumped charge through a single energy level during a cycle at zero temperature can be calculated [7] using emissivity [35]

$$
Q=\frac{e}{\pi} \int_{A} \mathrm{~d} \lambda_{1} \mathrm{~d} \lambda_{2} \operatorname{Im}\left(\frac{\partial s_{11}^{*}}{\partial \lambda_{1}} \frac{\partial s_{11}}{\partial \lambda_{2}}+\frac{\partial s_{12}^{*}}{\partial \lambda_{1}} \frac{\partial s_{12}}{\partial \lambda_{2}}\right),
$$

where $s_{\alpha \beta}$ is the scattering matrix element, and $\alpha$ and $\beta$ denote contacts 1 and 2 . During an adiabatic cycle

\footnotetext{
*corresponding author, eyang@venus.korea.ac.kr
}

the parameters $\lambda_{1}$ and $\lambda_{2}$, which control the shape of the dot, follow a closed path $C$ in the parameter space and the area defined by it is denoted as $A$, see Fig 1 .

The integration over the area $A$ suggests that quantum pumping depends on the geometric properties of the path and is independent on detailed time evolution. This indicates that quantum pumping in nano-semiconductors may be a manifestation of a matrix (non-Abelian) Berry phase [36, 37]. Similar issues have been investigated in quite different physical systems, such as superconducting circuits [38]. Recent investigations have shown that several semiconductor dots possess non-Abelian matrix Berry phases. Semiconductor excitons 39 and p-type electrons [40, 41] can exhibit matrix Berry phases. It has also been shown that n-type semiconductor dots with spin-orbit coupling possess a matrix (non-Abelian) Berry phase 42, 43, 44]. This is due to the presence of energy level degeneracy originating from time-reversal symmetry.

We show in this paper that adiabatic pumping in semiconductor quantum dots without spin-orbit coupling may be analyzed in terms of non-Abelian Berry phases. For non-Abelian Berry phases to be finite it is essential that degenerate states are present, and semiconductor quantum pumps do have non-trivial degenerate scattering states, see Fig 2] Each energy level of a quantum dot pump is doubly degenerate since there are two independent scattering states incident from the left and right barriers. During the pumping action these degenerate states can get mixed. This can be seen as follows: Let $T(t+\delta t, t)$ be the time development operator between $t+\delta t$ and $t$, and let the change of the confinement potential of the dot during the pumping action be $\delta V(t)=V(t+\delta t)-V(t)$, where $\delta t$ is an infinitely small time interval. The degenerate instantaneous eigenstates of the Hamiltonian are chosen as the degenerate scattering states $\left|\psi_{i}(t)\right\rangle$, shown in Fig 2. Suppose that the initial electron at $t$ is in the scattering state $\left|\psi_{1}(t)\right\rangle$. Then the overlap matrix element of the time development operator between the degenerate scattering eigenstates of the Hamiltonian is given by

$$
\begin{array}{r}
\left\langle\psi_{2}(t+\delta t, t)|T(t+\delta t, t)| \psi_{1}(t)\right\rangle \approx \\
\frac{i \delta t}{\hbar}\left\langle\psi_{2}(t)|\delta V(t)| \psi_{1}(t)\right\rangle
\end{array}
$$

since $T(t+\delta t, t) \approx 1-\frac{i}{\hbar} \delta t H(t)$. For some pumping ac- 
tions, given by Eq.(19), this overlap is finite, and the state $\left|\psi_{1}(t)\right\rangle$ can develop into a linear combination of $\left|\psi_{1}(t+\delta t)\right\rangle$ and $\left|\psi_{1}(t+\delta t)\right\rangle$. This suggests that during the adiabatic cycle a state vector may rotate in the degenerate Hilbert subspace and the initial state may not return to itself after the adiabatic cycle: $|\Psi(0)\rangle \neq|\Psi(T)\rangle$, as shown in Fig, It is unclear under what conditions this happens and how different the initial and final states are. Do they differ by a phase factor or more than a phase factor? Theory of non-Abelian matrix Berry phases [36] is well suited to address these issues.

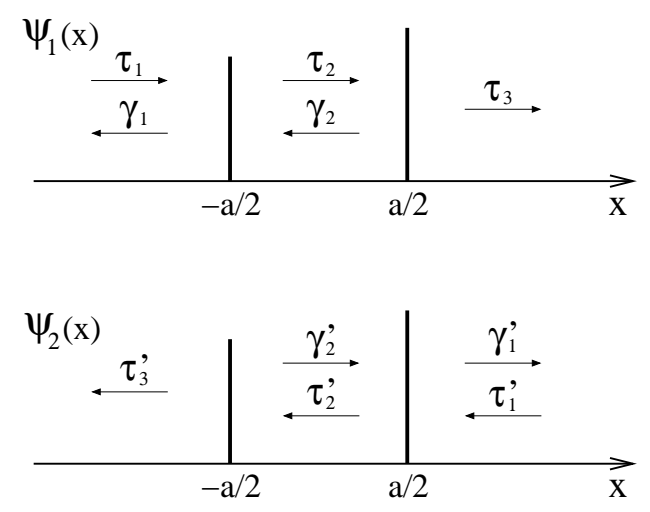

FIG. 2: In our model an electron moves in a one-dimensional asymmetric double barrier system without spin-orbit coupling. Two degenerate scattering states $\psi_{1}(x)$ and $\psi_{2}(x)$ are shown. $\tau_{i}, \tau_{i}^{\prime}$ and $\gamma_{i}, \gamma_{i}^{\prime}$ are wavefunction amplitudes.

We show that the presence of a finite matrix Berry phase leads to a non-zero pumped charge. Our investigation of time evolution of the electron state shows that the pumped charge depends on the initial state of the adiabatic cycle, i.e., different initial states give different pumped charges. Our method is applicable even when the potential modulations are substantial.

\section{NON-ABELIAN U(2) GAUGE THEORY, MATRIX BERRY PHASE, AND PUMPED CHARGE}

Non-Abelian Berry phases 36 are computed from the exact solutions of the time-dependent Schrödinger equation for adiabatic changes. In this approach one can choose the orthonormal basis states of the degenerate energy level as the instantaneous eigenstates of the Hamiltonian: $H(t)\left|\Phi_{i}(t)\right\rangle=E(t)\left|\Phi_{i}(t)\right\rangle$ for $i=1,2$. (Here we consider only adiabatic changes and other states with different energies are ignored). Even when the electron state starts in one of the instantaneous eigenstates $\left|\Phi_{i}(0)\right\rangle$ it may not stay in the same eigenstate $\left|\Phi_{i}(t)\right\rangle$ at later times: the actual state at $t$ may be a linear combination of the instantaneous eigenstates

$$
|\Psi(t)\rangle=c_{1}(t)\left|\Phi_{1}(t)\right\rangle+c_{2}(t)\left|\Phi_{2}(t)\right\rangle .
$$

For a cyclic change with the period $T$, the instantaneous basis states $\left|\Phi_{1}(T)\right\rangle$ and $\left|\Phi_{2}(T)\right\rangle$ return to the initial states $\left|\Phi_{1}(0)\right\rangle$ and $\left|\Phi_{2}(0)\right\rangle$, but the coefficients $c_{1}(T)$ and $c_{2}(T)$ may not return to the initial values. In this case, the initial and final degenerate states may be connected via a finite matrix Berry phase. A matrix Berry phase is a non-perturbative concept and is applicable even in the presence of strong potential modulations that are adiabatic. A matrix Berry phase can arise when an energy level has a non-trivial degeneracy which does not split during an adiabatic change of the external parameters $\lambda_{p}$ of the Hamiltonian. For a doubly degenerate energy level a matrix Berry phase is a $2 \times 2$ matrix $\Phi_{C}$ connecting the final amplitudes $\left(c_{1}(T), c_{2}(T)\right)$ to initial amplitudes $\left(c_{1}(0), c_{2}(0)\right)$ :

$$
\left(\begin{array}{c}
c_{1}(T) \\
c_{2}(T)
\end{array}\right)=\Phi_{C}\left(\begin{array}{l}
c_{1}(0) \\
c_{2}(0)
\end{array}\right) .
$$

Using the instantaneous degenerate basis vectors the matrix Berry phase can be computed by solving the adiabatic version of the time-dependent Schrödinger equation

$$
i \hbar \dot{c}_{i}=-\sum_{j} A_{i j} c_{j} \quad i=1,2,
$$

where $A_{i j}=\hbar \sum_{p}\left(A_{p}\right)_{i, j} \frac{d \lambda_{p}}{d t}$ and the sum over $p$ in $A_{i j}$ is meant to be the sum over $\lambda_{p}$. The non-Abelian gauge potentials are defined as

$$
\left(A_{p}\right)_{i, j}=i\left\langle\Phi_{i} \mid \frac{\partial \Phi_{j}}{\partial \lambda_{p}}\right\rangle .
$$

Solving the time-dependent Schrödinger equation, Eq.(5), one can write the matrix Berry phase in terms of the non-Abelian gauge potentials [36]:

$$
\begin{aligned}
\Phi_{C} & =e^{i \sum_{p} A_{p}\left(t_{n}\right) d \lambda_{p}} \ldots . . e^{i \sum_{p} A_{p}\left(t_{1}\right) d \lambda_{p}} \\
& =P e^{i \oint_{C} \sum_{p} A_{p} d \lambda_{p}}
\end{aligned}
$$

where a path ordering $P$ must be used since $A_{p}$ at different times are usually noncommuting. We see from this contour integration that

1. Matrix Berry phase is a geometric effect, i.e. it depends only on the path C.

2. Matrix Berry phase is independent of the period of the cycle as long as the change is adiabatic.

It is instructive to compare this result to the Abelian Aharonov-Bohm phase of an electron in the electromagnetic vector potential $\vec{A}$ :

$$
\Phi_{C}=e^{i \oint_{C} \vec{A} \cdot d \vec{\ell}}
$$

where $C$ is a closed path encircling the magnetic flux. The only difference between the Abelian and non-Abelian 
phases is the mathematical nature of the vector potentials: a number vs. matrix. Under a unitary transformation $\left|\psi_{i}^{\prime}\right\rangle=\sum_{j} U_{i j}^{*}\left|\psi_{j}\right\rangle$ the non-Abelian gauge structure emerges

$$
A_{k}^{\prime}=U A_{k} U^{\dagger}+i U \frac{\partial U^{\dagger}}{\partial \lambda_{k}} .
$$

For doubly degenerate levels the relevant gauge group is $U(2)$.

A pumped charge can be computed from the solution of the time-dependent Schrödinger equation, Eq.(15). It can be calculated from the total probabilities in the regions to the left, right, and center of the dot, denoted by

$$
\begin{aligned}
& P_{\mathrm{L}}(t)=\int_{-L}^{-a / 2}|\Psi(x, t)|^{2} \mathrm{~d} x, \\
& P_{\mathrm{R}}(t)=\int_{a / 2}^{L}|\Psi(x, t)|^{2} \mathrm{~d} x, \\
& P_{\mathrm{C}}(t)=\int_{-a / 2}^{a / 2}|\Psi(x, t)|^{2} \mathrm{~d} x,
\end{aligned}
$$

where $L$ is the length of the system. The change of these probabilities after the $n$th adiabatic cycle, $t=n T$, is

$$
\Delta P_{\mathrm{i}}(n)=P_{\mathrm{i}}(n)-P_{\mathrm{i}}(n-1) .
$$

for $i=L, C, R$. The probabilities $\Delta P_{L}$ and $\Delta P_{R}$ are directly related to the pumped charge: the pumped charge after the first cycle is

$$
Q=\Delta P_{L}(1)=-\Delta P_{R}(1),
$$

provided that the change of probability in the dot, $\Delta P_{C}(1)$, is negligible. It is straightforward to relate the matrix Berry phase to the pumped charge using Eqs. (4) and (12). The dependence on the non-Abelian Berry phases enters through Eq.(4), and the pumped charge depends on the initial conditions $\left(c_{1}(0), c_{2}(0)\right)$ and basis states $\Phi_{j}(x)$ :

$$
\begin{aligned}
Q & =\sum_{i j} O_{i j}\left[c_{i}(T)^{*} c_{j}(T)-c_{i}(0)^{*} c_{j}(0)\right] \\
& =\sum_{i j k l} O_{i j}\left[\left(\Phi_{C}\right)_{i k}^{*}\left(\Phi_{C}\right)_{j l}-\delta_{i k} \delta_{j l}\right] c_{k}(0)^{*} c_{l}(0),
\end{aligned}
$$

where

$$
O_{i j}=\int_{-L}^{-a / 2} \Phi_{i}(x)^{*} \Phi_{j}(x) d x
$$

\section{MODEL CALCULATION OF PUMPED CHARGE}

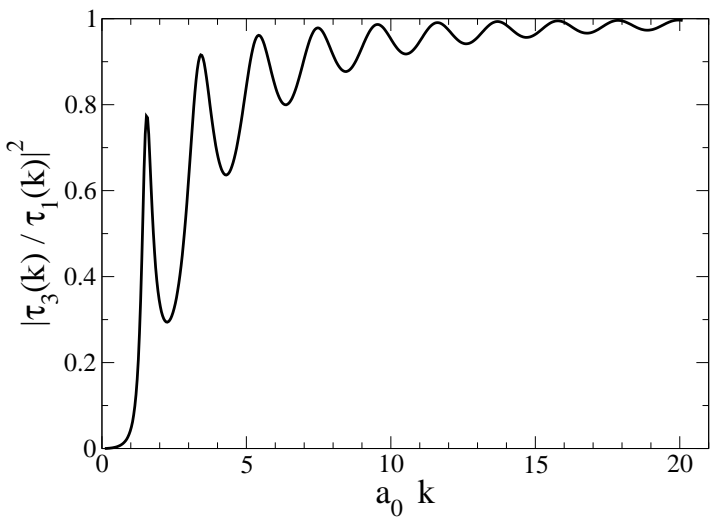

FIG. 3: Transmission coefficient of a double barrier system with the parameters $V_{l} /\left(E_{0} a_{0}\right)=4.25$ and $V_{r} /\left(E_{0} a_{0}\right)=2.0$.

The simplest possible model to study quantum pumping is a one-dimensional double barrier system [45, 46]. If the barrier potentials are chosen to be delta functions it is possible to calculate analytically the eigenstates and the non-Abelian vector potentials. Let us consider two delta-function barriers with the distance $a$ :

$$
V(x)=V_{l} \delta\left(x+\frac{a}{2}\right)+V_{r} \delta\left(x-\frac{a}{2}\right),
$$

where $V_{l}$ and $V_{r}$ are heights of the left and right barriers, respectively. Each energy level is doubly degenerate with eigenstates $\psi_{1}(x)$ and $\psi_{2}(x)$ : in $\psi_{1}(x)\left(\psi_{2}(x)\right)$ the electron is incident from the left (right), see Fig.1. Due to time reversal invariance the scattering amplitudes satisfy $\tau_{3} / \tau_{1}=\tau_{3}^{\prime} / \tau_{1}^{\prime}$. In the following we consider the pumped charge of an electron in the energy level $E=\frac{\hbar^{2} k^{2}}{2 m}$, where $k$ is a wavevector and $m$ is the electron effective mass. We use the energy unit $E_{0}=\frac{\hbar^{2}}{2 m a_{0}^{2}}$ and the length unit $a_{0}=2 a / 3$.

In the numerical calculation of the pumped charge it is convenient to use box normalization with length $L$. Let us comment on how the limit $L \rightarrow \infty$ is taken. It is natural to choose the incoming scattering states from the left and right as the instantaneous basis states: $\psi_{1}(x)$ and $\psi_{2}(x)$ (any other orthonormal set can also be used to calculate the non-Abelian vector potentials). However, for a finite value of $L$ these two states are not orthogonal. We choose one of the instantaneous basis state as $\psi_{1}(x)$ and construct the other basis state via Gram-Schmidt orthogonalization using $\psi_{2}(x)$ :

$$
\begin{aligned}
& \Phi_{1}(x)=\psi_{1}(x), \\
& \Phi_{2}(x)=\alpha \psi_{1}(x)+\beta \psi_{2}(x),
\end{aligned}
$$

As $L \rightarrow \infty$ the overlap $\left\langle\psi_{1} \mid \psi_{2}\right\rangle \rightarrow 0$ and $\Phi_{2}(x) \rightarrow \psi_{2}(x)$. Below the pumped charge is calculated for sufficiently large values $L$ where the size dependence is negligible. 


\section{A. Parity symmetry: absence of matrix Berry phase and pumped charge}

When the double barrier potential retains parity symmetry during the adiabatic cycle, i.e. $V_{l}(t)=V_{r}(t)$, the pumped charge is zero. (The adiabatic parameters are $\left.\left(V_{l}(t), a(t)\right)\right)$. This follows from the expression for the pumped charge given in Eq.(11): we have verified that the imaginary part of $\frac{\partial s_{11}^{*}}{\partial V_{l}} \frac{\partial s_{11}}{\partial a}+\frac{\partial s_{12}^{*}}{\partial V_{l}} \frac{\partial s_{12}}{\partial a}$ is zero. We show that this is fully consistent with the absence of a matrix Berry phase. For this purpose it is convenient to choose the following orthonormal instantaneous basis states:

$$
\begin{aligned}
& \Phi_{1}(x)=\frac{1}{\sqrt{N_{1}}}\left(\psi_{1}(x)+\psi_{2}(x)\right), \\
& \Phi_{2}(x)=\frac{1}{\sqrt{N_{2}}}\left(\psi_{1}(x)-\psi_{2}(x)\right),
\end{aligned}
$$

where the normalization factors are $N_{1}=2+2\left\langle\psi_{1} \mid \psi_{2}\right\rangle$ and $N_{2}=2-2\left\langle\psi_{1} \mid \psi_{2}\right\rangle$. We will use this orthonormal set to calculate the non-Abelian vector potentials 47 . Since two barrier heights are equal, i.e. $V_{l}=V_{r}$, it can be easily shown that $\psi_{1}(x)=\psi_{2}(-x)$. From this it follows that $\Phi_{1}(x)$ and $\Phi_{2}(x)$ are, respectively, even and odd functions in $x$. Taking the above states as basis vectors, and using the results $\left\langle\psi_{1}\left|\frac{\partial}{\partial \lambda_{p}}\right| \psi_{1}\right\rangle=\left\langle\psi_{2}\left|\frac{\partial}{\partial \lambda_{p}}\right| \psi_{2}\right\rangle$ and $\left\langle\psi_{1}\left|\frac{\partial}{\partial \lambda p}\right| \psi_{2}\right\rangle=\left\langle\psi_{2}\left|\frac{\partial}{\partial \lambda p}\right| \psi_{1}\right\rangle$ with the orthogonality $\left\langle\Phi_{1} \mid \Phi_{2}\right\rangle=0$ we find that the following off-diagonal elements of vector potentials are zero:

$$
\begin{aligned}
\left(A_{p}\right)_{12}= & i\left\langle\Phi_{1} \mid \frac{\partial \Phi_{2}}{\partial \lambda_{p}}\right\rangle \\
= & \frac{i}{\sqrt{N_{1}^{*} N_{2}}}\left(\left\langle\psi_{1}\left|\frac{\partial}{\partial \lambda_{p}}\right| \psi_{1}\right\rangle+\left\langle\psi_{1}\left|\frac{\partial}{\partial \lambda_{p}}\right| \psi_{2}\right\rangle\right. \\
& \left.-\left\langle\psi_{2}\left|\frac{\partial}{\partial \lambda_{p}}\right| \psi_{1}\right\rangle-\left\langle\psi_{2}\left|\frac{\partial}{\partial \lambda_{p}}\right| \psi_{2}\right\rangle\right)=0 .
\end{aligned}
$$

Similarly $\left(A_{p}\right)_{21}=0$ can be proved. Since the offdiagonal elements of the vector potentials are zero the matrix Berry phase is zero. This effect of parity symmetry is already known from the investigation of matrix Berry phases of isolated II-VI and III-V quantum dots in the presence of spin-orbit coupling: when the in-plane confinement potential of the dot has parity symmetry the matrix Berry phase vanishes [42, 43].

\section{B. Numerical calculation of pumped charge}

When the parity symmetry is broken during the adiabatic cycle, i.e. $V_{l}(t) \neq V_{r}(t)$, a pumped charge is expected. We choose the adiabatic parameters as $V_{l}$ and $V_{r}$ and select elliptic paths

$$
\begin{aligned}
& \left(V_{l}(t), V_{r}(t)\right)= \\
& \left(V_{l, c}+\Delta V_{l} \cos (\omega t), V_{r, c}+\Delta V_{r} \sin (\omega t)\right) .
\end{aligned}
$$

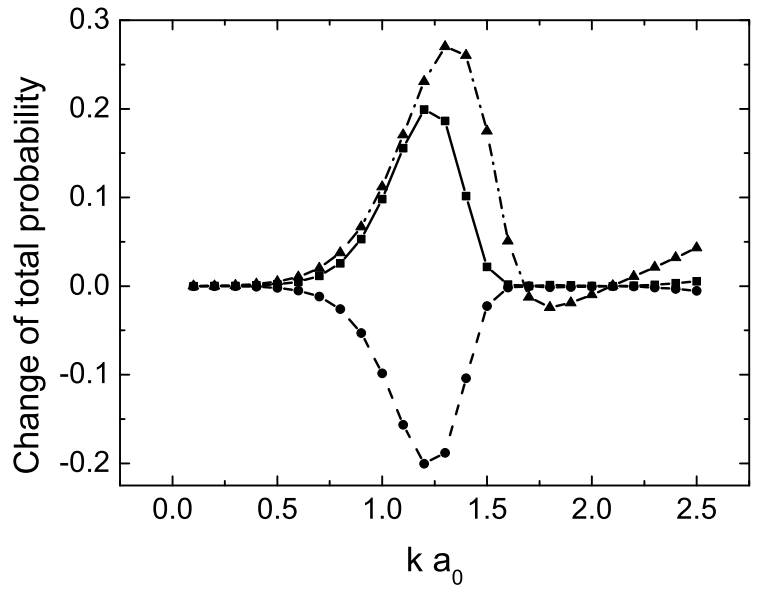

FIG. 4: Numerical results for the change in the total probabilities in different space regions after the first adiabatic cycle as a function of wavevector: $\Delta P_{\mathrm{L}}(1)$ (squares) and $\Delta P_{\mathrm{R}}(1)$ (circles). The pumped charge $Q / e$ calculated analytically using Eq.(1) (triangles). We have used $L / a_{0}=500$ and $\omega / E_{0}=0.1$.

At $t=0$ we start with the scattering state $\left|\psi_{1}\right\rangle$. We investigate pumped charges when the potential modulations are substantial : $\Delta V_{l} / V_{l, c}=0.7, \Delta V_{r} / V_{r, c}=0.7$, where $V_{l, c} /\left(a_{0} E_{0}\right)=2.5$ and $V_{r, c} /\left(a_{0} E_{0}\right)=2.0$. At the start of the adiabatic cycle the quantum dot is characterized by the scattering amplitudes shown in Fig 3 . The time dependent Schrödinger equation, Eq.(5) is solved numerically, and we determine the amplitudes $c_{1,2}(T)$, from which the matrix Berry phase can be computed using Eq.(4). The pumped charge after the first cycle, $\Delta P_{\mathrm{L}}(1)$, is calculated as a function of wavevector using Eqs.(10). The results are shown in Fig 4, The pumped charge is maximum near $k a_{0}=1.25$, which is close to the position of the first resonance in the transmission coefficient, $k a_{0}=1.56[45,46]$. Note that the vanished probability on the right side of the dot appears on the left side: $\Delta P_{\mathrm{L}}(1)=-\Delta P_{\mathrm{R}}(1)$. This implies that the pumped charge does not accumulate in the dot, which is consistent with Eq.(11), see Ref.[7]. We have also compared quantitatively our results for the pumped charges with those obtained from Eq.(1): they are in a good qualitative agreement.

In our approach we can follow microscopically how various quantities actually depend on time. Fig 5 displays time dependence of the total probabilities in different space regions during consecutive adiabatic transformations. Results are shown for $k a_{0}=1.30$. We note that $P_{L}(n)$ for $n=0,1, \ldots$ are all different, implying that pumped charge $\Delta P_{L}(n)$ depends on the number of consecutive adiabatic cycles. This is because initial state of the $n$th cycle $\Psi((n-1) T)$ is different from that of the $(n-1)$ th cycle $\Psi((n-2) T)$. In Eq.(1) this dependence 


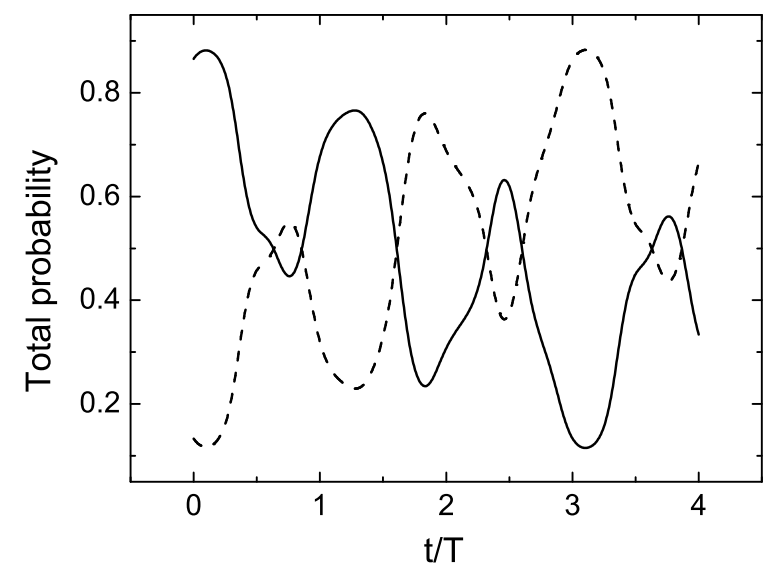

FIG. 5: Time dependence of the total probabilities in different space regions, $P_{\mathrm{L}}$ (solid) and $P_{\mathrm{R}}$ (dashed), during consecutive adiabatic transformations.

on initial states is not present. We believe it is because the initial states are chosen to be some particular scattering states, see, for example, Eq.(12) of Ref.[12]. Note that the probability fluxes through the left and right barriers are equal to each other at all times: $\frac{d P_{L}}{d t}=-\frac{d P_{R}}{d t}$. During the period of a cycle the instantaneous pumped current displays a significant variation in time.

\section{CONCLUSIONS}

Solving the time dependent Schrödinger equation exactly for adiabatic changes we find that the initial and final states are connected via a finite matrix Berry phase. This difference between initial and final states of an adiabatic cycle implies that a pumped charge is present. The main physics behind this is that even when the electron state starts in one of the instantaneous eigenstates it may not stay in the same eigenstate at later times: often the actual state at time $t$ turns out to be a linear combination of the instantaneous eigenstates. One can prepare the initial state to be the incident scattering state from the left. When consecutive adiabatic cycles are performed starting from this initial state the pumped charge at the $n$th cycle is not necessarily the same as that of the $(n-1)$ th cycle. Our investigation of the time dependence thus shows that the pumped charge depends on the nature of the initial state of a cycle. Moreover, during the period of a cycle the probability flux through the dot displays a significant variation in time. It would be interesting to test these results experimentally.

\section{Acknowledgments}

S.R.E.Y thanks M.Governale for useful discussions. He is also grateful to R.Fazio for reading this manuscript and bringing Ref.[38] to his attention. This work was supported by grant No. R01-2005-000-10352-0 from the Basic Research Program of the Korea Science and Engineering Foundation and by Quantum Functional Semiconductor Research Center (QSRC) at Dongguk University of the Korea Science and Engineering Foundation. This work was supported by The Second Brain Korea 21 Project.
[1] D.J. Thouless, Phys. Rev. B 27, 6083 (1983).

[2] B. L. Altschuler and L. I. Glazman, Science 283, 1864 (1999).

[3] M. Switkes, C. M. Marcus, K. Campman and A. C. Gossard, Science 283, 1905 (1999).

[4] L.P.Kouwenhoven, A. T. Johnson, N. C. vanderVaart, C. J. P. M. Harmans, C. T. Foxon, Phys. Rev. Lett. 67, 1626 (1991).

[5] H. Pothier, P. Lafarge, C. Urbina, D. Esteve and M. H. Devoret, Europhys. Lett 17, 249 (1992).

[6] S. K. Watson, R.M. Potok, C.M. Marcus, and V. Umansky, Phys. Rev. Lett.91, 258301 (2003).

[7] P. W. Brouwer, Phys. Rev. B 58, R10135 (1998).

[8] F. Zhou, B. Spivak and B. Altshuler, Phys. Rev. Lett. 82, 608 (1999).

[9] T.A. Shutenko, I.L. Aleiner, and B.L. Altshuler, Phys. Rev. B 61, 366 (2000).

[10] A. Andreev and A. Kamenev, Phys. Rev. Lett. 85, 1294 (2000).
[11] I.L. Aleiner, B.L. Altshuler, and A. Kamenev, Phys. Rev. B 62, 10373 (2000).

[12] O. Entin-Wohlman, A. Aharony and Y. Levinson, Phys. Rev. B 65, 195411 (2002).

[13] J. E. Avron, A. Elgart, G. M. Graf and L. Sadun, Phys. Rev. B 62, R10618 (2000).

[14] H.-Q. Zhou, S. Y. Cho, and R. H. McKenzie, Phys. Rev. Lett.91, 186803 (2003).

[15] Y. Makhlin and A. D. Mirlin, Phys. Rev. Lett.87, 276803 (2001).

[16] Y. Levinson, O. Entin-Wohlman and P. Wolfle, Physica A 302, 335 (2001).

[17] L.E.F. Foa Torres, Phys. Rev. B 72, 245339 (2005).

[18] A. Agarwal and D. Sen, J. Phys. Condens. Matter 19, 046205 (2007).

[19] I. Sela and D. Cohen, J. Phys. A: Math. Gen. 39, 3575 (2006).

[20] M. V. Berry and J. M. Robbins, Proc. R. Soc. Lond. A 442, 659 (1993). 
[21] L. S. Levitov, arXiv:cond-mat/0103617

[22] M. Moskalets and M. Büttiker, Phys. Rev. B 66, 035306 (2002).

[23] M. Moskalets and M. Büttiker, Phys. Rev. B 64, 201305(R) (2001).

[24] M.L. Polianski, M. G. Vavilov and P.W. Brouwer, Phys. Rev. B 65, 245314 (2002).

[25] I. L. Aleiner and A. V. Andreev, Phys. Rev. Lett. 81, 1286 (1998)

[26] P. W. Brouwer, A. Lamacraft, and K. Flensberg, Phys. Rev. B 72, 075316 (2005).

[27] T. Aono, Phys. Rev. Lett. 93, 116601 (2004).

[28] R. Citro, N. Andrei, and Q. Niu, Phys. Rev. B 68, 165312 (2003)

[29] J. Splettstoesser, M. Governale, J. König, and R. Fazio, Phys. Rev. B 74, 085305 (2006)

[30] P. Sharma and C. Chamon, Phys. Rev. Lett. 87, 96401 (2001)

[31] A. Agarwal and D. Sen, Phys. Rev.B 76,035308(2007).

[32] E. R. Mucciolo, C. Chamon, and C. M. Marcus, Phys. Rev. Lett. 89, 146802 (2002)

[33] M. Governale, F. Taddei, and R. Fazio, Phys. Rev. B 68, 155324 (2003)

[34] M. Blaauboer, Phys. Rev. B 68, 205316 (2003).

[35] M. Büttiker, H. Thomas, and A. Prêtre, Z. Phys. B, 94,
133 (1994).

[36] F. Wilczek and A. Zee, Phys. Rev. Lett. 52, 2111 (1984);

[37] Geometric Phases in Physics, edited by A. Shapere and F. Wilczek (World Scientific, Singapore, 1989).

[38] V. Brosco, R. Fazio, F.W.J. Hekking, and A. Joye, arXiv:cond-mat/0702333.

[39] P.Solinas, P. Zanardi, N. Zanghi, F. Rossi, Phys. Rev. A 67, 062315 (2003).

[40] Yu. A. Serebrennikov, Phys. Rev. B 70, 064422 (2004).

[41] B. A. Bernevig and S.-C. Zhang, Phys. Rev. B 71, 035303 (2005).

[42] S.-R. Eric Yang and N.Y. Hwang, Phys. Rev. B 73, 125330 (2006).

[43] S.-R. Eric Yang, Phys. Rev. B 74, 075315 (2006).

[44] S.-R. Eric Yang, Phys. Rev. B 75, 245328 (2007).

[45] M. Blaauboer and E. J. Heller, Phys. Rev. B 64, 241301(R) (2001).

[46] Y. Wei, J. Wang, and H. Guo, Phys. Rev. B 62, 9947 (2000).

[47] As long as a degenerate pair of basis states can be related to another pair by a unitary transformation the nonAbelian gauge structure, Eq. (99), is satisfied, see Ref. [36]. This is true independent of any model. 